\title{
Prevention of Surgical Site Infection in Pediatric Reconstructive Surgery under Bone Marrow Suppression
}

\author{
Yohei Hayashi ${ }^{1 *}$, Makoto Mihara ${ }^{1 *}$, Takuya lida ${ }^{1 *}$, Hisashi Moriguchi ${ }^{1}$, Rintaro Hirai ${ }^{1}$, Azusa Oshima $^{1}$, \\ Mitsunaga Narushima', Isao Koshima ${ }^{1}$ \\ 1:Department of Plastic Surgery and Reconstructive Surgery, The University of Tokyo, Japan
}

*These authors contributed equally

received 2010.5.12 accsepted 2010.5.24

\section{A bstract}

Surgical site infection (SSI) is the most common infection following a surgical procedure and reduction of SSI is an important issue for health professionals and patients. In particular, SSI may develop into a serious infection in cancer patients with decreased white blood cell counts due to chemotherapy and in pediatric patients with a weak immune system. Because SSI often occurs through sutures used in surgery that remain in the body, improvement of sutures has been used as a countermeasure against SSI. In 2003, the introduction of a suture containing an antibacterial agent opened a new avenue to prevention of SSI. In this study, we report a case of pediatric malignant rhabdoid tumor in which head reconstructive surgery was performed using the antibacterial suture with successful control of infectious diseases. We also provide an overview of the current situation and perspectives on prevention of SSI.

\section{K e y w o r d s Supermicrosurgery, Vicryl Plus, Testicular cryopreservation}

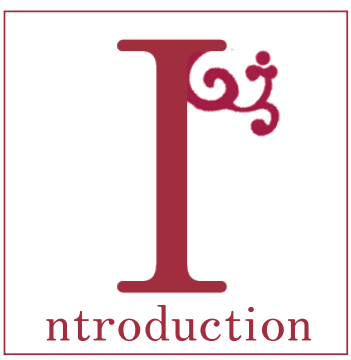

Introduction

Surgical site infection (SSI) is the most frequent postoperative infection, accounting for about $38 \%$ of hospital infections. SSI is a medical concern due to the increased risk of complications and has an economic effect through prolongation of the period of hospitalization. The occurrence of SSI is broadly due to background factors such as pathogens carried innately by patients, or to operative factors such as infection from operative instruments. Therefore, risk management for SSI with consideration of these factors is an important issue in reduction of medical and economic burdens.

Relationships between SSI and surgical suture

Since over $60 \%$ of SSI is estimated to occur at incision sites, the most important operative factor in SSI appears to be surgical sutures. These sutures remain in the body after surgery and may become footholds for infection of pathogens. To reduce the rate of SSI caused by sutures, 
a synthetic suture that is absorbed in the body after surgery was developed in the 1970s. Although this suture reduced SSI, it did not resolve the problem of attachment of pathogens to the suture before absorption. Subsequently, the monofilament suture was developed to reduce facilitation of infection compared to the earlier twiny suture, but this change also failed to produce a sufficient reduction in SSI.

\section{Development of an antibacterial suture}

Antibacterial sutures (PLUS Antibacterial Sutures; Ethicon, Inc.), in which an antibacterial agent is mixed with the coating agent, were introduced in 2003 for fundamental prevention of SSI. In one such suture (the Coated VICRYL Plus Suture; Irgacare MP) very high purity triclosan is used as the antibacterial agent ${ }^{1)}$. In vitro, these sutures significantly inhibit colony formation of the most common surgical site pathogens, including methicillin-resistant Staphylococcus aureus (MRSA) and Staphylococcus epidermidis, compared to sutures without antibacterial agents ${ }^{2}$. Such antibacterial sutures are particularly helpful for cancer patients with decreased white blood cell counts due to chemotherapy, and in patients with weak immune systems, such as infants and the elderly. Here, we report a case of pediatric malignant rhabdoid tumor in which we used the Coated VICRYL Plus Suture in head reconstructive surgery and had a positive outcome for inhibition of SSI.

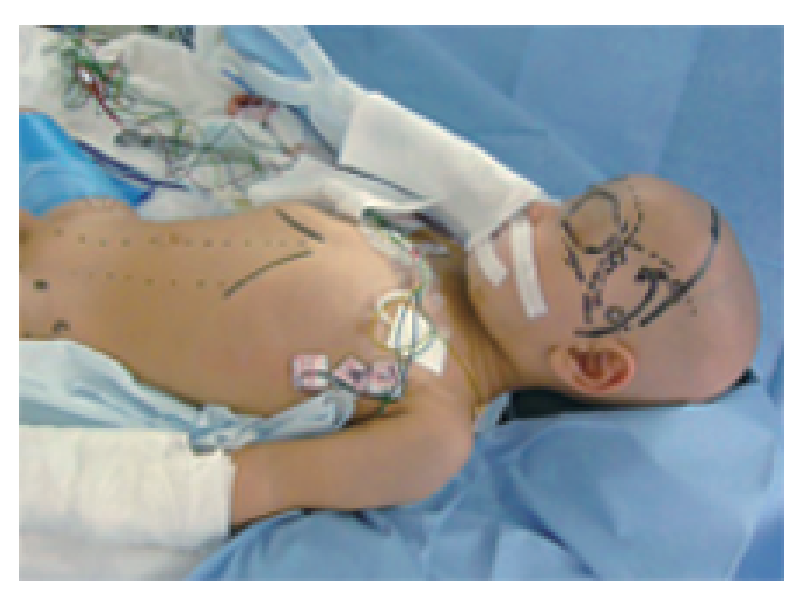

Figure 1.

Perioperative findings. Shaving of hair with an electric shaver and sterilization with povidone iodine were performed before the surgery for prevention of infection.

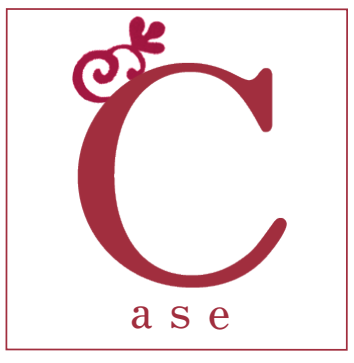

Case

The patient was a male infant aged one year old (Figure 1). He was $72.8 \mathrm{~cm}$ tall and weighed 7.2 $\mathrm{kg}$, was eugenic, and had no apparent neural symptoms. He was born in November 2008 with a birth weight of $3348 \mathrm{~g}$ after 39 weeks and 3 days gestation. A tumor mass was visible in the left eye at birth and showed a tendency to increase. In January 2009, laser therapy and a local injection of steroids were administered in the Department of Plastic Surgery at Gunma Children's Medical Center due to suspicion of angioma. However, these therapies did not resolve the tendency for tumor growth. In February 2009, a biopsy was performed in the Department of Pediatrics at the center, with a resulting diagnosis of rhabdoid tumor and lung metastasis. The tumor was reduced in size and the lung metastasis disappeared after a course of chemotherapy using "high-risk rhabdomyosarcoma protocol 8". In September 2009, autologous stem cell transplantation and supermass chemotherapy were performed, and MRI in December 2009 showed elimination of tumor growth and metastasis.

The surgery included resection of the enlarged skull base tumor (Figure 2), enucleation of the eye, transplantation of a free rectus abdominis musculocutaneous flap (Figure $3 \mathrm{~A})$, and dural reconstruction with transplantation of the deep fascia of the thigh.

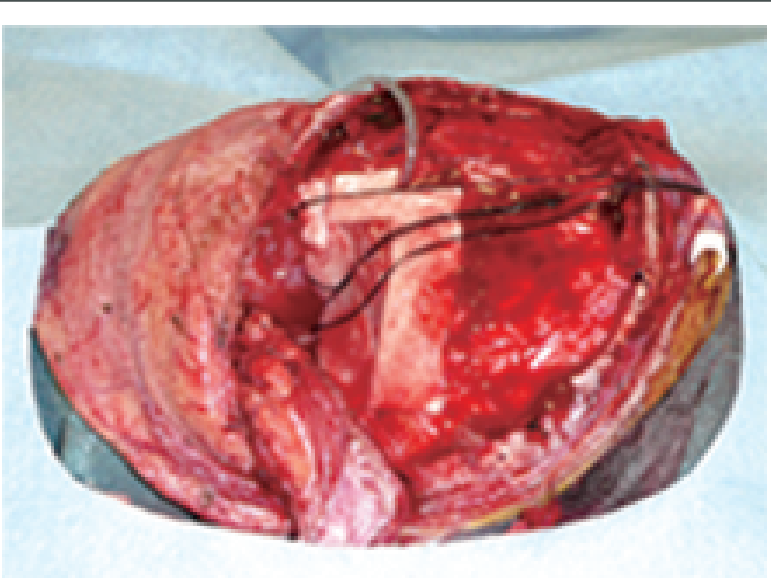

Figure 2.

Photographs of the site after tumor resection. The resection site in the dura mater was closed by transplantation of the deep fascia of the thigh. 
The operative time was $14 \mathrm{~h}$ and $21 \mathrm{~min}$, with blood infusion level of $440 \mathrm{ml}$, a volume of infusion of $555 \mathrm{ml}$, and a hemorrhage level during transplantation of $230 \mathrm{ml}$. Suture for the anterior sheath of the rectus abdominis muscle, abdominal wall subcutaneous suture, and head subcutaneous suture were performed using Vicryl Plus (total number of sutures: approx. 350) (Figure 3B). Closed suction drainage was performed with 6 drains, including 2 head subcutaneous sites, the left femoral subfascial site, the left abdominal subfascial site, and the tumorectomy site, and 1 cisternal drain. Cephem antibiotics were administered 3 times during surgery (0.14 g each time), and drip infusion of these antibiotics was performed 3 times a day (also $0.14 \mathrm{~g}$ each time) for 9 days after surgery. The postoperative course was unremarkable, with no observation of surgical site infection (Figure 4), suture abscess, or systemic infection (Table 1).
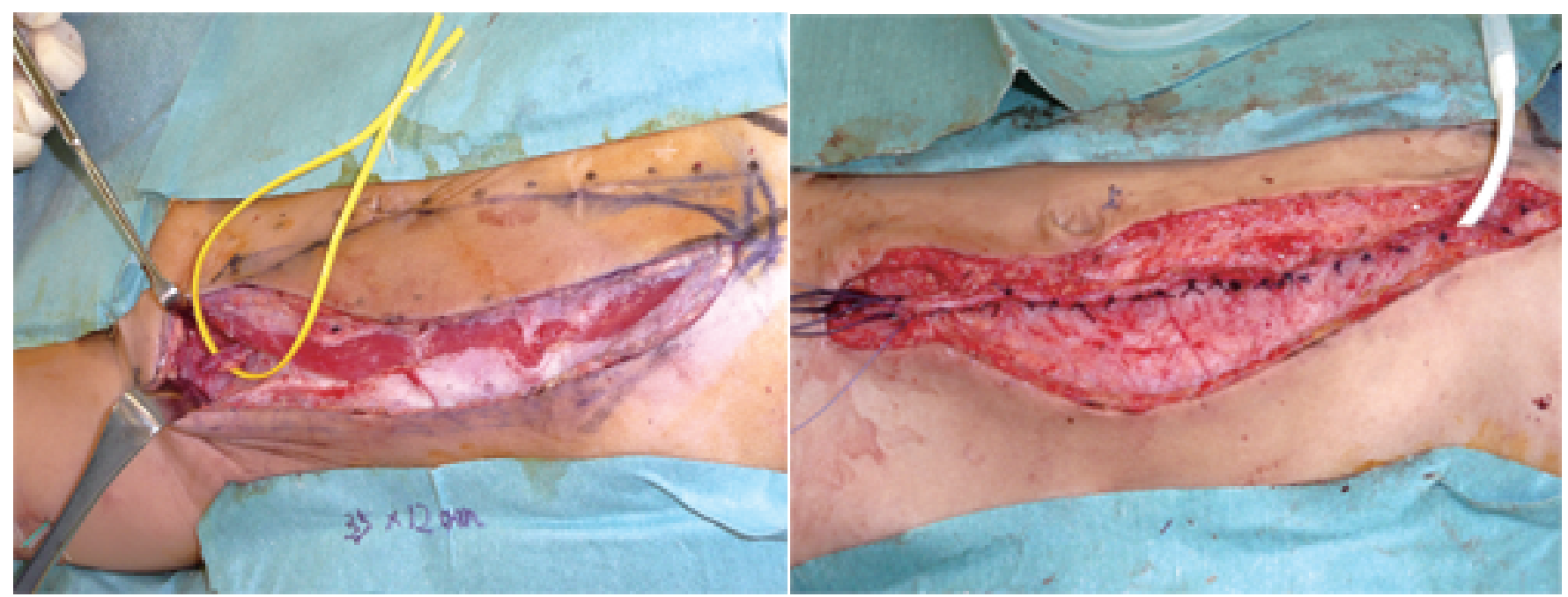

Figure 3.

(A) Removal of the rectus abdominis muscle. About $80 \%$ of the rectus abdominis muscle was used.

(B) The anterior sheath of the rectus abdominis muscle was sutured using Vicryl Plus.

Table 1. Changes in blood test results

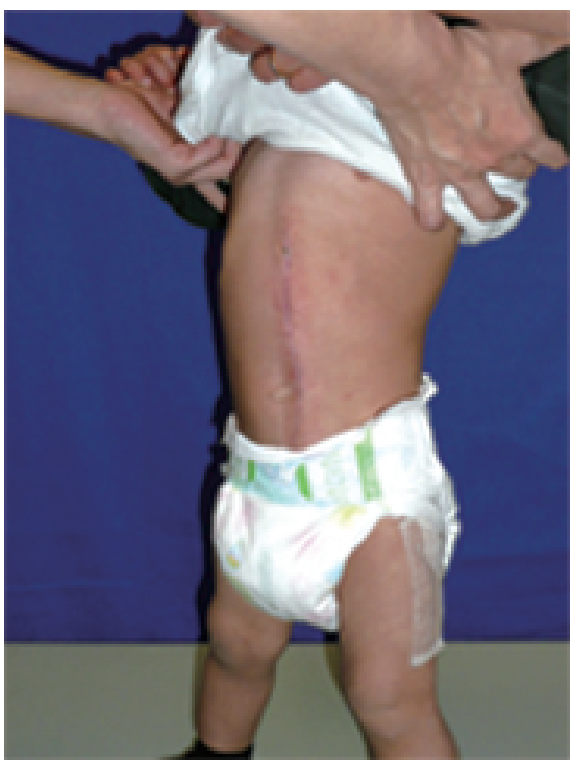

Figure 4 .

Subcutaneous abscess, surgical site infection, and abdominal wall hernia were not observed at 1 month after the surgery.

\begin{tabular}{|l|c|c|c|c|}
\hline Data* & WBC & Hb & PLT & CRP \\
\hline 2010.01.08. 13:32 & 2.6 & 8.6 & 16.5 & 0.32 \\
\hline \multicolumn{5}{|c|}{ Operation } \\
\hline 2010.01.13. 01:08 & 5.8 & 10.3 & 9.1 & 1.13 \\
\hline 2010.01.13. 06:30 & 8.4 & 11.1 & 10.9 & 5,14 \\
\hline 2010.01.14. 07:17 & 10.4 & 9.9 & 10.8 & 17.39 \\
\hline 2010.01.15. 07:31 & 8.4 & 9.1 & 9.9 & 9.99 \\
\hline 2010.01.16. 06:47 & 4.6 & 11.3 & 8.1 & 3.90 \\
\hline 2010.01.17. 07:35 & 6.1 & 11.3 & 9.2 & 2.61 \\
\hline 2010.01.18. 06:15 & 5.0 & 12.1 & 10.1 & 2.24 \\
\hline 2010.01.19. 08:12 & 4.4 & 12.2 & 10.3 & 1.51 \\
\hline 2010.01.20. 10:05 & 4.5 & 12.5 & 14.5 & 0.96 \\
\hline 2010.01.25. 10:47 & 5.4 & 13.1 & 17.4 & 0.20 \\
\hline
\end{tabular}

*WBC: white blood cells, Hb: hemoglobin, PLT: platelets, CRP: C-reactive protein 


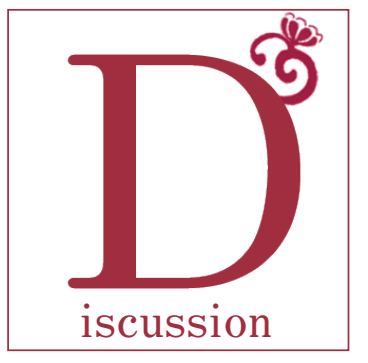

Discussion

In this study, we used a Coated VICRYL Plus antibacterial suture for transplantation of the free rectus muscle in a patient with a pediatric malignant rhabdoid tumor. The procedure was performed with successful inhibition of postoperative infection, which indicates the effectiveness of the antibacterial suture in a pediatric patient with a weakened immune system due to medication with anticancer drugs. Inhibition of SSI is a critical aspect of reconstructive surgery for patients after bone marrow suppression by chemotherapy. Since over $60 \%$ of SSI occurs at incision sites, the inhibition of spread of infection from these sites is particularly important. In this regard, the use of antibacterial sutures is desirable compared with non-antibacterial sutures and administration of antibiotics. Antibacterial sutures also allow focused delivery of antibacterial agents to incision sites, thereby contributing to a reduction of side effects at other sites.

From an economic perspective, the Coated VICRYL Plus suture is about $15-20 \%$ more expensive than non-antibacterial VICRYL sutures, but it is far cheaper when the costs for an extended hospital stay due to SSI infection are included ${ }^{3}$. In addition, since the treatment of SSI is included in the operation costs in comprehensive healthcare costs in Japan, the development of SSI inflicts heavy losses on a hospital. Therefore, risk reduction through use of antibacterial sutures can substantially contribute to the medical economy, and further development of these sutures is desirable.

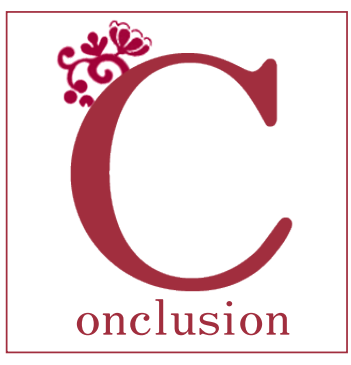

\section{Conclusion}

The Coated VICRYL Plus suture used in this surgery is based on the novel idea of addition of an antibacterial agent to a synthetic absorbable suture. The effectiveness of the antibacterial suture has been proven by in vitro inhibition of bacterial colonization on the suture ${ }^{2)}$ and in vivo in animal experiments ${ }^{4)}$. The clinical utility of the suture has been shown in cardiovascular surgery (2007), neurosurgery (2008) and digestive surgery (2009). However, as indicated below, further accumulation of cases, including information on drug disposition, is required to establish the efficacy of this suture.

Issue 1: Collection of drug disposition data

There are few clinical findings on the disposition of drugs from antibacterial sutures, including the length of time and extent to which the antibacterial agent in the suture persists in the body. Collection of these data would provide important information.

Issue 2: Differences between adults and children There may be differences between adults and children in the optimum concentration of antibacterial agents and the absorption efficiency of absorbable sutures. Development of antibacterial sutures suitable for children requires collection of pediatric clinical data.

\section{Issue 3: Development of multiagent sutures}

The activity of an antibacterial suture clearly depends on the type of antibacterial agents in the suture. The versatility of antibacterial sutures could be expanded by inclusion of multiple antibacterial agents in one suture for growth inhibition of all bacterial strains. 


\section{References}

1) Gilbert P, Mcbain AJ. Literature-based evaluation of the potential risks associated with impregnation of medical devices and implants with triclosan. Surg Infect. 2002;3 Supple 1:S55-S63.

2) Edmiston CE, Seabrook GR, Goheen MP, et al. Bacterial adherence to surgical sutures: can antibacterial-coated sutures reduce the risk of microbial contamination? J Am Coll Surg.

2006;203(4):481-9

3) Fleck T, Moidl R, Blacky A, et al. Triclosan-coated sutures for the reduction of sternal wound infections: economic considerations. Ann Thorac Surg. 2007;84(1):232-6.

4) Marco F, Vallez R, Gonzalez P, et al. Study of the efficacy of coated Vicryl plus antibacterial suture in an animal model of orthopedic surgery. Surg Infect.

2007;8(3):359-65.

\section{Web References}

* Ethicon, Inc.

http://www.ethicon.com/

\section{Acknowledgements}

This study was supported by grants from the following organizations. The authors would like to thank them all: - Japan Society for the Promotion of Science (Grants-in-Aid for Young Scientists A)

- Japan Interaction in Science and Technology Forum Foundation

- Leave a nest Co., Ltd. (Leave a nest Grant)

- Foundation for Promotion of Cancer Research

- Children's Cancer Association of Japan

- Fukuoka University, School of Medicine Alumni, Eboshikai

- Mitsui Life Social Welfare Foundation

- National Center for Child Health and Development 